\title{
The paths from research to improved health outcomes
}

$\mathrm{E}$ vidence-based practice aims to provide clinicians and patients with choices about the most effective care based on the best available research evidence. To patients, this is a natural expectation. To clinicians, this is a near impossible dream. The US report Bridging the quality chasm has documented and drawn attention to the gap between what we know and what we do. ${ }^{1}$ The report identified 3 types of quality problems-overuse, underuse, and misuse. It suggested "The burden of harm conveyed by the collective impact of all of our healthcare quality problems is staggering." Although attention has focused on misuse (or error), a larger portion of the preventable burden is likely to be the evidence-practice gaps of underuse and overuse.

Research that should change practice is often ignored for years-for example, crystalloid (rather than colloid) for shock, ${ }^{2}$ supine position after lumbar puncture, ${ }^{3}$ bed rest for any medical condition, ${ }^{3}$ and appropriate use of anticoagulants and aspirin in patients with atrial fibrillation. ${ }^{4}$ Antman et al documented the substantial delays between cardiovascular trial results and textbook recommendations. ${ }^{5}$ However, even when best practices are well known, they are often poorly implemented: national surveys show that most hypertensive patients are undetected, untreated, or inadequately controlled, ${ }^{6}$ which has led to the current interest in knowledge translation. ${ }^{7}$

\section{PRACTICE FAMINE AMIDST THE EVIDENCE GLUT}

What role does evidence-based practice ${ }^{8}$ have in bridging the research-practice gap? Surveys of clinicians suggest that a major barrier to using current research evidence is the time, effort, and skills needed to access the right information among the massive volumes of research. ${ }^{9}$ Even for a (mythical) up to date clinician, the problem of maintaining currency is immense. Each year Medline indexes >560 000 new articles, and Cochrane Central adds about 20000 new randomised trials. This is about 1500 new articles and 55 new trials per day! Clinicians need clear and efficient strategies to sift, digest, and act on new research likely to benefit their patients. Two stages can be considered: getting the evidence straight and getting the straight evidence used. ${ }^{10}{ }^{11}$

\section{Getting the evidence straight}

Although individual new research articles are peer reviewed and published, there is little effort to set their results systematically in the context of other, similar studies. ${ }^{12}$ Ideally, clinicians could access an updated, well conducted systematic review for all questions, or at least for all clinical research. However, only about $10 \%$ of randomised trials have currently been incorporated into Cochrane systematic reviews. ${ }^{13}$ For non-treatment questions, the situation is worse. Guidelines are not a panacea here, as they usually rely on existing reviews, or, more often, ignore evidence, ${ }^{14}$ and are rarely presented in clinician friendly formats. Hence the Institute of Medicine report recommended that we "establish and maintain a comprehensive programme aimed at making scientific evidence more useful and accessible to clinicians and patients."

\section{Getting the evidence used}

Clinicians frequently have questions about the care of their patients, but most go unanswered. ${ }^{15}$ Even when questions are "answered," it is often by using out of date textbooks within the immediate clinical setting. The main predictors of the attempt to answer a question are the belief that an answer exists and the urgency of the patient's problem. ${ }^{16}$

This lack of bedside use of evidence inspired the 4 step model of bedside evidence-based practice: ${ }^{8}{ }^{17}$ (i) ask an answerable question; (ii) track down the best evidence; (iii) critically appraise the evidence for validity, impact, and applicability; and (iv) integrate the results with the patient's unique biology, circumstances, and values. In teaching evidence-based practice, integration of the steps into the clinical setting and for real patient problems is crucial for changing attitudes and behaviour. ${ }^{18}$ Following these steps in clinical practice is challenging, especially given time constraints and the skills needed to complete these steps.

\section{THE EVIDENCE PIPELINE}

What underlies substantial gaps between the best evidence and the management patients receive? Pathman et al ${ }^{19}$ described 4 stages from evidence to action: the clinician needs to be aware, then agree, then adopt, and then adhere. Their survey of physicians' use of vaccine guidelines showed a steady decline at each stage-for example, the rates for acellular pertussis were $90 \%$ aware, $67 \%$ agree, $46 \%$ adopt, and $35 \%$ adhere. This is consistent with findings from research on the diffusion of innovations, ${ }^{20}$ which generally suggest a 5 stage model of knowing, accepting, deciding, implementing, and continuing. A subsequent systematic review of barriers to the use of evidence ${ }^{9}$ suggested that several further stages might be added. The figure extends the awareness-to-adherence model to include these newer elements-in particular, patient involvement.

The model illustrates that, even with high rates of transfer between stages, there may be little impact on patient outcomes. Thus, even $80 \%$ transfer at each of 7 stages would result in only a $21 \%$ patient usage $\left(0.8^{7}=0.21\right)$.

Using this model, we shall look, firstly, at the initial problem of getting the valid and relevant evidence into the clinical "pipelines" and how this can be improved, and secondly, at methods for reducing blockages at each stage.

\section{Awareness}

Given our information glut, it is not surprising that individual clinicians find it difficult to be aware of all the relevant, valid evidence. Profitable new interventions are likely to have a substantial marketing campaign. However, for many important practice changes, such as low cost pharmaceuticals or non-pharmaceuticals, awareness is more problematic. To ease the burden, several scanning and alert services have arisen that help clinicians become aware of important changes. For example, journals such as EvidenceBased Nursing and Evidence-Based Medicine scan $>100$ journals to identify new evidence that is valid and important, and this process has been augmented to build a new service, bmjupdates ${ }^{+}$(http://bmjupdates.com), that allows 


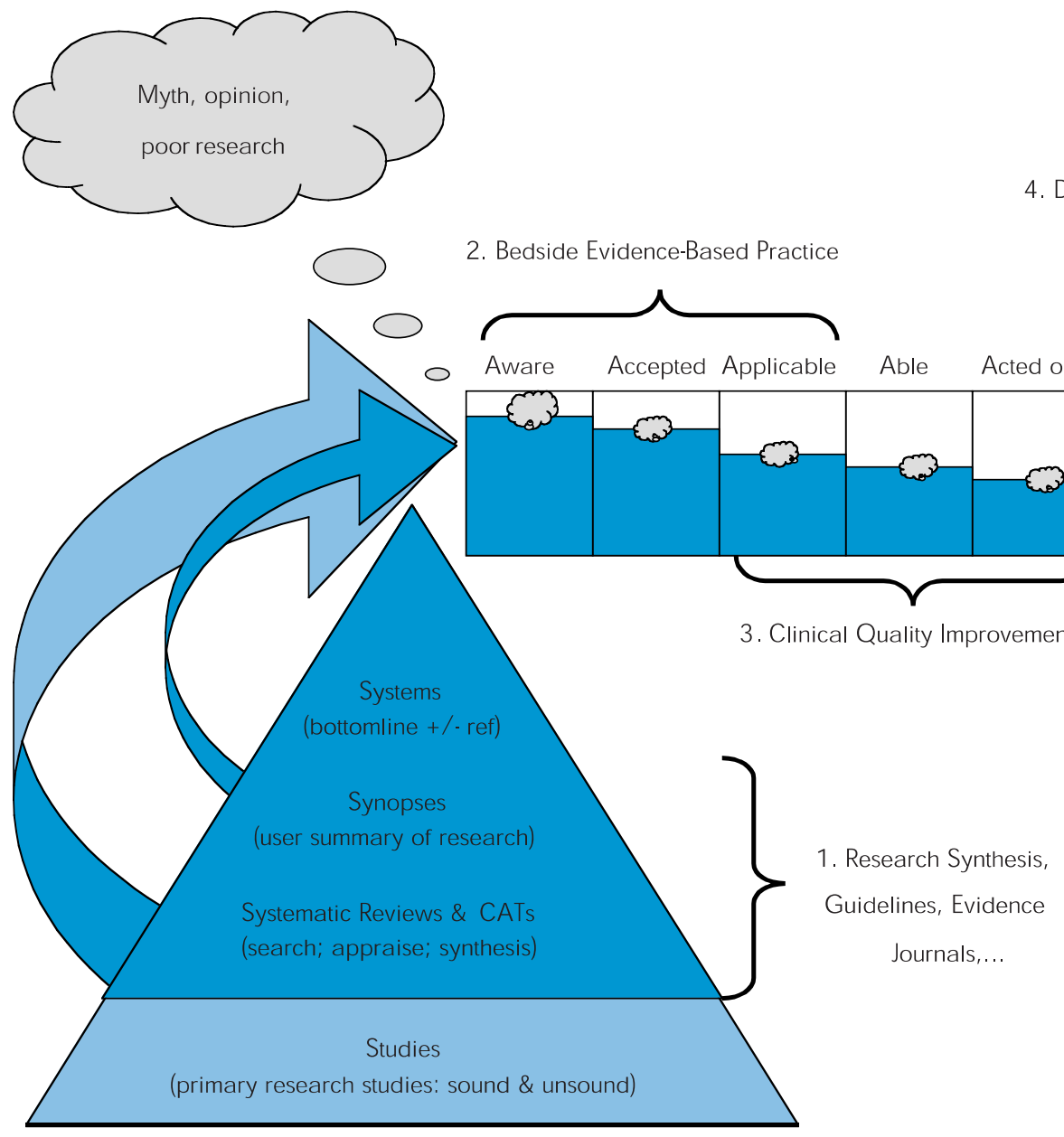

The research-to-practice pipeline. New research, of varying soundness, is added to the expanding pool and enters practice both directly or is reviewed, summarised, and systematised (delay) before entering practice, with leakage occurring at each of several stages between awareness and patient outcome. Different knowledge translation disciplines focus on different parts of the pipeline (1-4).

practitioners to tap into just those articles that their physician peers rate as highly relevant and interesting for clinical practice (see p39 for more information about this new free service).

\section{Acceptance}

Although practitioners may have heard of the benefits of a new intervention or the harms of an old one, they may not be persuaded to change management based on this evidence. A central problem is that clinicians may be persuaded by means other than unbiased evidence, such as the marketing techniques of advertising, reciprocity (the obligation arising from "gifts"), authority, social validation (acceptance by peers), and friendship/personal relationships. ${ }^{21} 22$ Pharmaceutical companies and others invest considerable resources in such methods. Hence more work is needed to identify methods that can best "vaccinate" clinicians against poor evidence.

\section{Applicable}

Even if evidence is accepted, clinicians and guidelines may not target the correct groups. For example, a review of 20 guidelines for atrial fibrillation (most of which were not evidence-based) showed that the proportion of patients recommended for warfarin varied from $13-100 \%{ }^{23}$ Whether there are net benefits of anticoagulation depends on balancing the risk of stroke against the risk of haemorrhage. A survey of doctors in Australia suggested good knowledge of factors that increased the haemorrhage risk, but only half correctly identified a patient with a previous stroke as being at high risk of stroke recurrence. ${ }^{24}$ Similarly, a Dutch study showed that risk factors that should predict a higher prescription rate of warfarin did not. ${ }^{25}$ Unfortunately, the relation between diagnosis and treatment is rarely one to one. Clinicians must usually learn about and understand the multiple factors that make good decisions that balance benefits and harms. ${ }^{26}$

\section{Available and able}

To carry out an intervention requires both access and knowhow. For medications, this is challenging enough: becoming familiar with dosing, contraindications, initiation, adverse effects, and monitoring. For more complex interventions such as brief counselling or spinal manipulation, the learning curve is even steeper and hence is a greater barrier to changing practice. For many complex interventions such as smoking cessation, external cephalic version, or problem solving therapy for depression, clinicians may require additional training before carrying these out as competently as in the trials that documented their benefits. 


\section{Acted on}

Even when we know and accept what to do, we often forget or neglect to do it. Habits do not change easily, despite our best intentions. Omissions are particularly easy for preventive procedures, as they are often not the pressing focus of a consultation. Not surprisingly, rates of appropriate preventive procedures are frequently low. A simple reminder is often sufficient for such simple omissions of interventions that we believe in and can do. A review of 16 randomised trials of reminders for preventive procedures showed substantial increases in adherence for most, but not all areas. ${ }^{27}$ Similar but less dramatic results have been shown for reminders in some areas of medication management..$^{28}$

\section{Agreed to}

When we have remembered to suggest an applicable treatment, the above steps may begin all over again for the patient. For the patient to agree, he/she must be aware of the options, accept that the management recommended is appropriate, be able to undertake it, etc. This may involve a complex mixture of the patient's values and beliefs, which thus need to be explored. To assist communication and understanding, patient decision aids have been developed. Although such aids can reduce patients' decisional conflict with their final choice, it is less clear whether aided decisions result in better patient outcomes. ${ }^{29}$

\section{Adhered to}

Patients must also contend with competing claims and advice, adverse effects or fear thereof, and sometimes lack of ability to pay for tests and treatments. If resources to inform prescribers of current best evidence are inadequate, they are woefully more so for patients, despite such pioneering efforts as DipEx (www.dipex.org/). Even when patients accept the benefits of treatment and wish to comply, they may not. We may all agree to exercise more, eat less, or stop smoking, but too few do. Even for medications, dosing frequency, pill size, and forgetfulness can all cause problems. Typical adherence rates for medications are $<50 \%$. Improving adherence to short courses of treatment is relatively easy, but enhancing adherence with long term regimens is more difficult. Helpful elements include information about the regimen, counselling about the importance of adherence and how to organise medication taking, reminders, rewards and recognition for patients' efforts to follow the regimen, and enlisting social support from family and friends. ${ }^{30}$

\section{CONCLUSIONS}

Even when most clinicians are aware of evidence, there may be little impact on quality of care without further attention to the other stages. However, we would see initial awareness (and discrimination) of high quality research as the first large hurdle. Although bedside evidence-based practice has focused on clinicians becoming aware of and accepting the best quality research, it is clearly important but insufficient. Not all clinicians will have or use the skills of bedside evidence-based practice, ${ }^{31}$ and even the well skilled will fail to implement intended changes fully. Hence evidence-based practice should not just be concerned with clinical content but also with the processes of changing care and systems of care.

\section{PAUL GLASZIOU, MBBS, PhD University of Oxford, Oxford, UK}

BRIAN HAYNES, MD, PhD McMaster University, Hamilton, Ontario, Canada

Acknowledgements: the authors would like to thank Iain Chalmers and Sharon Straus for their helpful comments.
A modified version of this Notebook originally appeared in Evidence-

\section{Based Medicine.}

1 Institute of Medicine. Crossing the quality chasm: a new health system for the 21 st century. Washington: Institute of Medicine, 2001.

2 Wilkes MM, Navickis RJ. Patient survival after human albumin administration. A meta-analysis of randomized, controlled trials. Ann Intern Med 2001;135:149-64.

3 Allen C, Glasziou P, Del Mar C. Bed rest: a potentially harmful treatment needing more careful evaluation. Lancet 1999;354:1229-33.

4 Brass LM, Krumholz HM, Scinto JD, et al. Warfarin use following ischemic stroke among Medicare patients with atrial fibrillation. Arch Intern Med 1998;158:2093-100

5 Antman EM, Lau J, Kupelnick B, et al. A comparison of results of metaanalyses of randomized control trials and recommendations of clinical experts. Treatments for myocardial infarction. JAMA 1992;268:240-8.

6 Haijar I, Kotchen TA. Trends in prevalence, awareness, treatment, and control of hypertension in the United States, 1988-2000. JAMA 2003;290:199-206.

7 Davis D, Evans M, Jadad A, et al. The case for knowledge translation: shortening the journey from evidence to effect. BMJ 2003;327:33-5.

8 Sackett DL, Straus SE, Richardson WS, et al. Evidence-based medicine: how to practice and teach EBM. Edinburgh: Churchill-Livingstone, 2000.

9 Cabana MD, Rand CS, Powe NR, ef al. Why don't physicians follow clinical practice guidelines? A framework for improvement. JAMA 1999:282:1458-65.

10 Haynes RB, Sackett DL, Gray JAM, et al. Transferring evidence from research into practice: 2. Getting the evidence straight. ACP J Club 1997;126(1):A14.

11 Gray JAM, Haynes RB, Sackett DL, et al. Transferring evidence from research into practice: 3. Developing evidence-based clinical policy. ACP J Club 1997;126(2):A14.

12 Clarke M, Alderson P, Chalmers I. Discussion sections in reports of controlled trials published in general medical journals. JAMA 2002;287:2799-801.

13 Mallett S, Clarke M. How many Cochrane reviews are needed to cover existing evidence on the effects of healthcare interventions? Evidence-Based Medicine 2003;8:100-1.

14 Shaneyfelt TM, Mayo-Smith MF, Rothwangl J. Are guidelines following guidelines? The methodological quality of clinical practice guidelines in the peer-reviewed medical literature. JAMA 1999:281:1900-5.

15 Dawes M, Sampson U. Knowledge management in clinical practice: a systematic review of information seeking behavior in physicians. Int J Med Inform 2003;71:9-15.

16 Gorman PN, Helfand M. Information seeking in primary care: how physicians choose which clinical questions to pursue and which to leave unanswered. Med Decis Making 1995;15:113-9.

17 Sackett DL, Straus SE. Finding and applying evidence during clinical rounds: the "evidence cart. " JAMA, 1998;280:1336-8.

18 Coomarasamy A, Khan KS. What's the evidence that postgraduate teaching in evidence based medicine changes anything? A systematic review. BMJ 2004;329:1017-9.

19 Pathman DE, Konrad TR, Freed GL, et al. The awareness-to-adherence model of the steps to clinical guidelines compliance. The case of pediatric vaccine recommendations. Med Care 1996;34:873-889.

20 Rogers EM. Diffusion of innovations. 5th ed. New York: Free Press, 2003.

21 Cialdini RB. Influence: the psychology of persuasion. New York: Quill, 1998.

22 Roughead EE, Harvey KJ, Gilbert AL. Commercial detailing techniques used by pharmaceutical representatives to influence prescribing. Aust N Z J Med 1998;28:306-10.

23 Thomson R, McElroy $H$, Sudlow M. Guidelines on anticoagulant treatment in atrial fibrillation in Great Britain: variation in content and implications for treatment. BMJ 1998;316:509-13.

24 Peterson GM, Boom K, Jackson SL, et al. Doctors' beliefs on the use of antithrombotic therapy in atrial fibrillation: identifying barriers to stroke prevention. Intern Med J 2002;32:15-23.

25 Rutten $\mathrm{FH}$, Hak E, Stalman WA, et al. Is treatment of atrial fibrillation in primary care based on thromboembolic risk assessment? Fam Pract 2003;20:16-21.

26 Hunink M, Glasziou P, Siegel J, et al. Decision making in health and medicine: integrating evidence and values. Cambridge: Cambridge University Press, 2001

27 Shea S, DuMouchel W, Bahamonde L. A meta-analysis of 16 randomized controlled trials to evaluate computer-based clinical reminder systems for preventive care in the ambulatory setting. J Am Med Inform Assoc 1996;3:399-409

28 Bennett JW, Glasziou PP. Computerised reminders and feedback in medication management: a systematic review of randomised controlled trials. Med J Aust 2003;178:217-22

29 O'Connor AM, Stacey D, Entwistle V, et al. Decision aids for people facing health treatment or screening decisions. Cochrane Database Syst Rev 2003;(3):CD001431.

30 Haynes RB, McDonald H, Garg AX, et al. Interventions for helping patients to follow prescriptions for medications. Cochrane Database Syst Rev 2002;(2):CD000011.

31 Straus SE, Green ML, Bell DS, for the Society of General Internal Medicine Evidence-Based Medicine Task Force, et al. Evaluating the teaching of evidence based medicine: conceptual framework. BMJ 2004;329:1029-32. 